\title{
Laparoscopic gastric plication: Technique and results
}

\author{
Murat Üstün \\ Department of General Surgery, Medistate Hospital, Istanbul, Turkey
}

\begin{abstract}
Laparoscopic gastric plication is a relatively new restrictive procedure. Promising early period results have been reported. When compared with other restrictive methods, excess weight loss at an acceptable level can be obtained at an early stage. Aim of this study was to discuss the technique and results obtained from 45 cases.
\end{abstract}

Keywords: Bariatric surgery; greater curvature plication; laparoscopic gastric plication.

\section{Introduction}

Morbid obesity is on the increase over the world, particularly in the West, at an epidemic level. ${ }^{[1]}$ The morbidity and mortality rates among these patients keep increasing compared to those with Normal BMI values. ${ }^{[2,3]}$ Since 1997, WHO has defined obesity as a global epidemic, and it was ascertained that there were more than 400 million obese people around the world in 2005.

Conservative methods such as diet, physical exercise and drug therapy have been proven to be insufficient. ${ }^{[4,5]}$ Many different methods that can provide significant and long-term weight loss have been developed in the last decade. ${ }^{[6,7]}$

During this period, an intense interest developed in restrictive methods such as adjustable gastric band and sleeve gastrectomy, among both bariatric surgeons and obese people. ${ }^{[8,9]}$ However, it has been asserted that gastric band is the least effective method compared to other commonly applied bariatric methods; and furthermore, requiring frequent postoperative adjustments as well as band slippages and erosion rates have been controversial subjects. ${ }^{[10,11]}$ As for sleeve gastrectomy, its irreversibility, cost, and most importantly leakage rates are still among controversial issues.

Laparoscopic gastric plication is a relatively new restrictive method born as a result of search for a safe, effective ( $>50 \% \mathrm{EWL}$ ), less invasive, reversible, repeatable, and economical method, with the thought that the ideal procedure for morbid obesity surgery has yet to be found. It was introduced first by Tretbar in 1976, and then by Wilkinson in 1981. ${ }^{[12,13]}$ In 1969, there was an experimental study describing the method.

The method that had been forgotten for a long time was suggested as a current issue again in 2006 by Talebpour who developed its technical details. ${ }^{[14]}$ In the experimental 
studies conducted in 2007, efficiencies of anterior plication and greater curvature plication were compared, and consequently, greater curvature plication was reported to be more efficient (Figure 1).

The method, applied to a series of large-volume cases by Talebpor for the first time, has since then been put into practice in various centers throughout the world.

A variety of names such as laparoscopic greater curvature plication (LGCP), gastric plication, gastric imbrication, and gastric folding have been offered for naming of the method.

The alleged advantages of the method include being an effective restrictive procedure like sleeve gastrectomy, a less complex operation, a reversible method that does not involve resection, and having minimal major complication risk and no staple line. It is based on preclinical data obtained in consequence of the previously performed animal experiments suggesting that the serosa-to-serosa sutures are permanent and reliable, and the weight loss is at a significant level and long-lasting.

\section{Materials and Methods}

A total of forty-five subjects, who underwent laparoscopic gastric plication between June 2010 and October 2012, were included into the study. Those who had previously undergone any bariatric surgery were excluded from the study. Their demographic data, complications and excess weight loss percentages were evaluated.

Thirty of the subjects were female (66\%) with a mean age of 32.5 (range, 18-49). BMI ranged from 35 to 50.8, and mean BMI was $41 \mathrm{~kg} / \mathrm{m}^{2}$. All subjects were informed about the surgical options and were asked to sign informed consent forms. Preoperative preparations specified in bariatric surgery protocol were carried out for all patients.



Figure 1.

\section{Surgical Procedure}

French and 30 degree reverse Trendelenburg position were studied on all patients. The abdomen was accessed by means of an optical trocar (Visiport), and pneumoperitoneum was formed in all patients again. Optical trocar was inserted $1-2 \mathrm{~cm}$ from midline and approximately $10-12$ $\mathrm{cm}$ from caudal of the xiphoid. The study was performed using a total of four trocars, which were inserted $5 \mathrm{~mm}$ from Subxiphoid (liver retractor), $10 \mathrm{~mm}$ from the left paramedian area (power device), and $5 \mathrm{~mm}$ from the right paramedian area. A fifth trocar was used in cases where the omentum was required to be excluded (Figure 2).

A greater curvature dissection was made from about 5-6 $\mathrm{cm}$ proximal of the pylorus, extending up to the esophagocardiac junction. Attention was paid to protect the left and right gastroepiploic arteries and His angle. First, a plication was made in a way so as to ensure that the vascular dissection line was in the middle, from the fundus to the antrum, by using $2 / 0$ nonabsorbable braided sutures one by one.

Then, the second layer of plication was made with $2 / 0$ nonabsorbable monofilament continuous suture. In the last twenty subjects, a continuous plication was achieved, using non-absorbable barbed sutures (V-Loc) in both layers. Again, in this second group, another system was begun to be used, in which the suture passed across four points, two of which were at the maximum distance in anterior and posterior and each of the others were close to the midline, as described by Talebpour (Figure 3). All sutures were passed extramucosally.

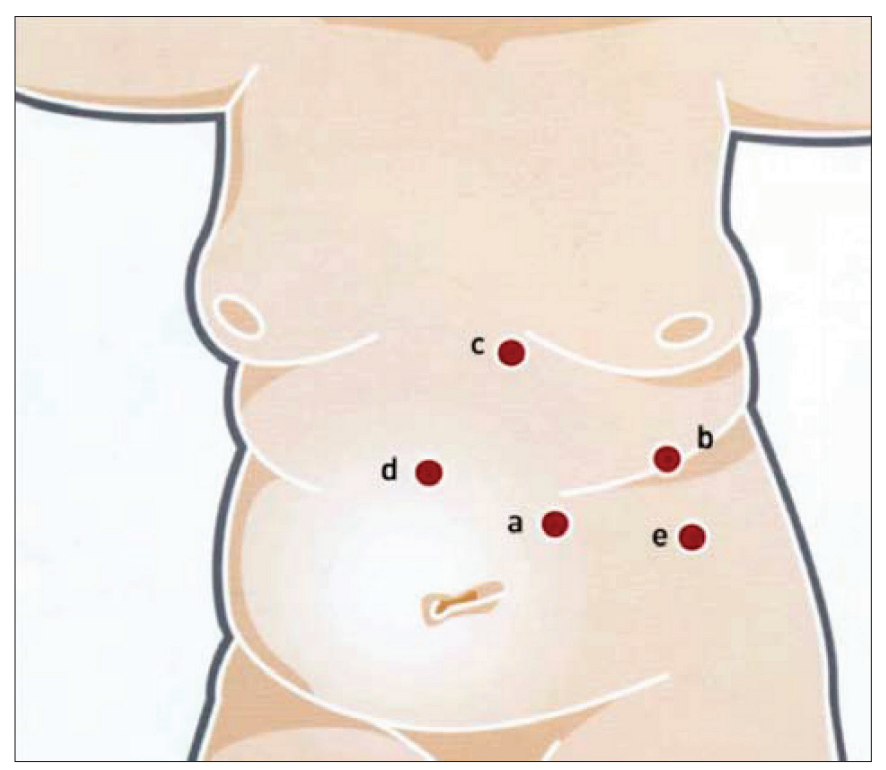

Figure 2. 


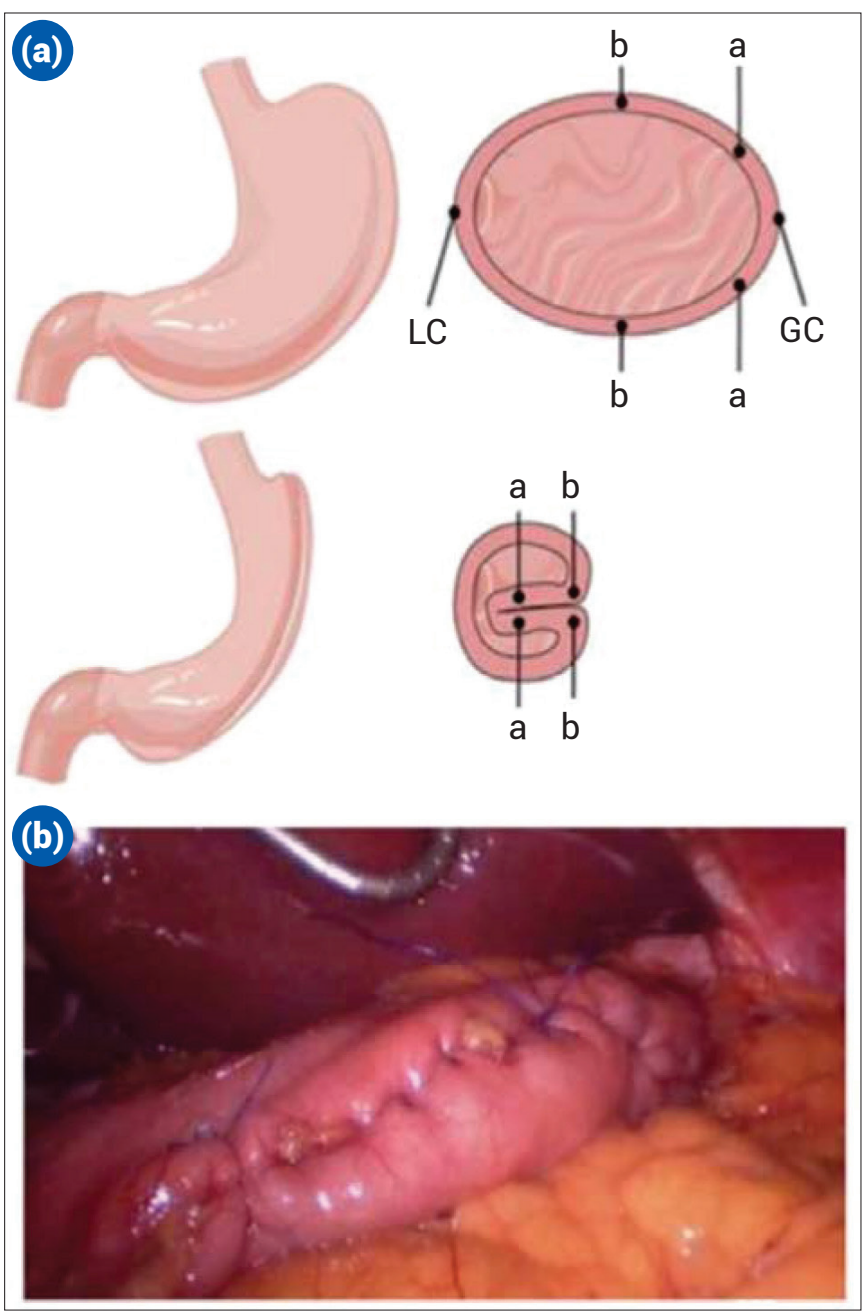

Figure 3.

During the operation, there were 36 French calibration tubes in the stomach. After the completion of the plication process, the pylorus was closed; and when the volume was controlled with a serum containing methylene blue, it was ascertained that a volume of about $60-100$ $\mathrm{mL}$ was left.

All patients were hospitalized until they relieved of nausea and vomiting, and began to orally intake adequate amount of liquid. During this period, proton pump inhibitors, prokinetic, antiemetic and antispasmodic medication and adequate fluid-electrolyte replacement were provided. On the second day, all patients got a bowel follow-through examination with a water soluble radiopaque substance, and when the bowels were observed to be normal, a liquid diet was started (Figure 4).

In the postoperative diet, a liquid diet was given for the first fifteen days, which was followed by a semi-solid mash diet given for the second fifteen days, as practiced in sleeve gastrectomy cases. The shortest patient follow-up

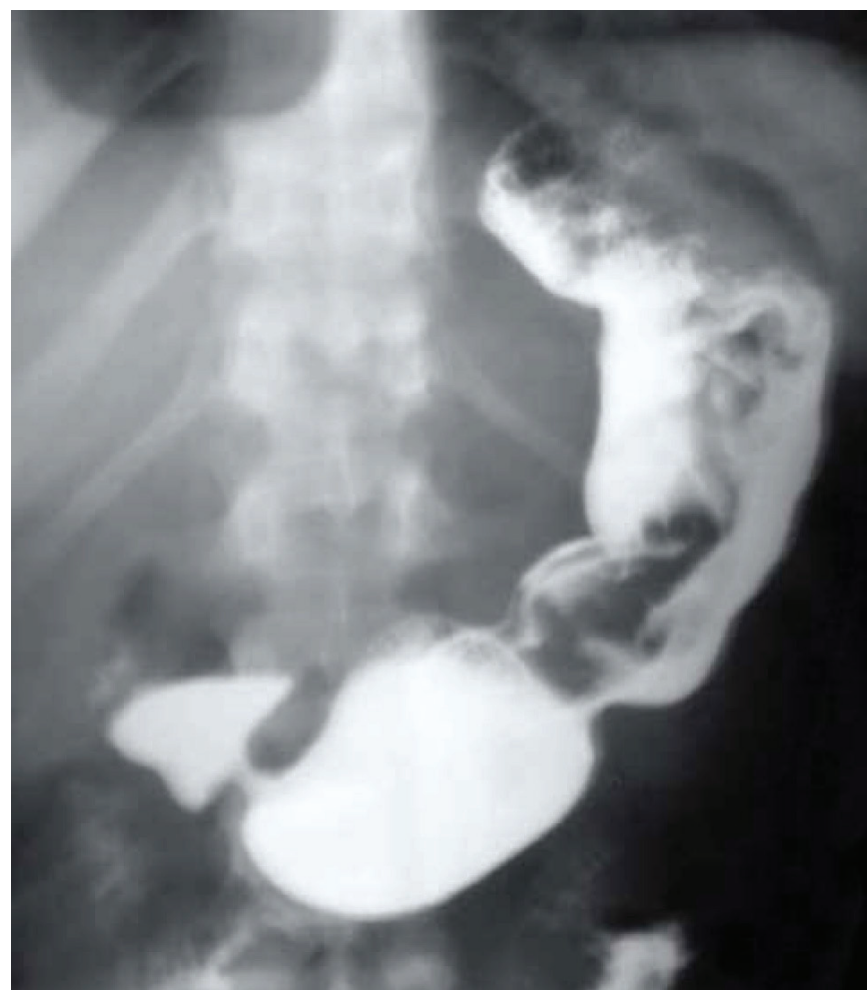

Figure 4.

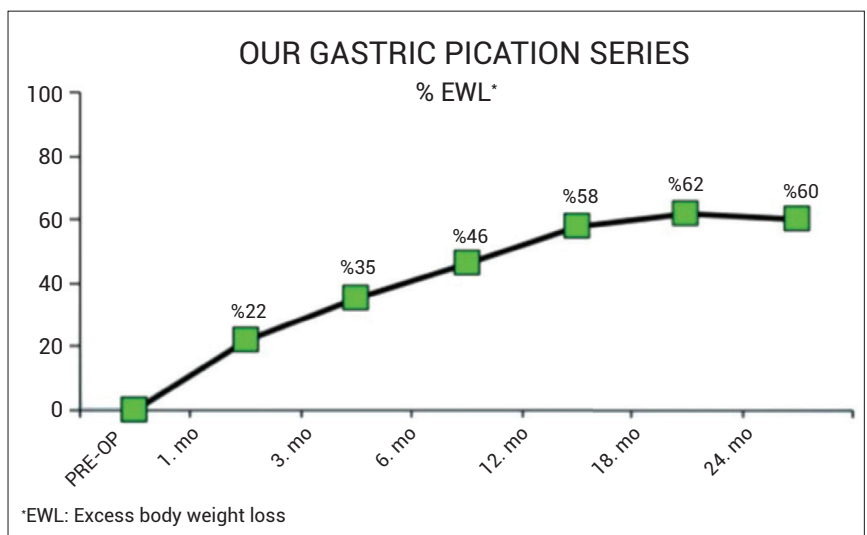

Figure 5.

period was six months, whereas the longest patient follow-up period was four years.

In the first month, an EWL (excess weight loss) up to $22 \%$ was observed in all patients. Operation time was 55-140 minutes, whereas mean operation time was 65 minutes. Hospitalization period was 24-96 hours (mean, 36 h). Return to daily life activities took 4-13 days (mean, 7 days) (Figure 5).

The most commonly encountered early complication was nausea, which was severe in $60 \%$ of the subjects, and it could not be relieved despite medication. Vomiting was the second severe complaint in $25 \%$ of the patients. Sialorrhea was observed in $20 \%$ of the subjects. As an intra- 




Figure 6.

operative complication, a trocar injury that led to mesentery bleeding in the small intestine developed in one subject (associated with the suture that passed through the abdomen during abdominoplasty). In that subject, an open surgery was started. The mortality rate was 0 .

\section{Early and Late Postoperative Complications}

In the first month, re-laparoscopy was required in two subjects due to persistent vomiting. Acute gastric obstruction was observed in the performed endoscopies. A subject was treated with deplication, and the other was treated with sleeve gastrectomy revision. The number of the patients requiring re-laparoscopy was one in the first 6 months, and deplication was done to the subject in whom a fundal herniation was observed during the tests performed after excessive vomiting associated with food intoxication (Figure 6). In the late period, sleeve gastrectomy revision was obtained by performing re-laparoscopy due to inadequate weight loss in a total of four subjects.

\section{Discussion}

Laparoscopic gastric plication is essentially an old but also a contemporary approach. Promising early-period results have been reported. When compared to other restrictive methods, excess weight loss at an acceptable level could be obtained at the early stage. Brethauer et al. have reported a significant decrease in appetite and a successful hunger control. ${ }^{[15]}$ However, re-hospitalization rates were relatively high at the early stage due to the high level of nausea and vomiting. ${ }^{[16]}$ Shen et al. have reported success rates lower than that of sleeve gastrectomy at the early stage..$^{[17]}$
El-Geider et al. have published the 4-bit technique recommended by Talebpour and described a firm plication technique defining a 4-needle passage at each suture, which begins $2 \mathrm{~cm}$ below the His angle. ${ }^{[18]}$

When compared to sleeve gastrectomy, the decrease in restrictive effect and weight re-gain are higher in the longterm. In these patients, it is somewhat hard to maintain weight for the long term. Probably, the causes of this situation are the fundus that cannot be resected and Ghrelin effect that cannot be utilized.

Only one publication is available about the long-term results of laparoscopic gastric plication, and weight re-gain was reported to be $15 \%$ (11 of 75 cases) in the third year; $30 \%$ (10 of 35 cases) in the seventh year; and 50\% (5 of 10 cases) in the tenth year. ${ }^{[19]}$ Again, in this study, the reluctance of the patient to participate in postoperative control and support groups has been found to be proportional to weight re-gain.

There is no study on the effect of postoperative follow-up on weight loss rates in the cases of laparoscopic gastric plication. However, Saunders and Ashton have mentioned about the positive effect of cognitive behavioral group therapy on patients in compulsive eater group in particular, who have undergone surgery. ${ }^{[2,21]}$

\section{Conclusion}

Laparoscopic gastric plication is a promising and relatively new method of restrictive bariatric surgery, reported to be able to provide acceptable EWL in well-motivated patients, whose BMI values are not over 45 . In the last 10 years, studies of this method have been carried out in many centers around the world. Its advantages are that it is partly reversible, it has a lower cost since it does not require stapler, it does not involve resection, and it poses a relatively lower risk of leakage, and shows a restrictive effect to an extent close to that of sleeve gastrectomy.

In our opinion, its most important disadvantages are that it causes a high rate of occurrence of early postoperative complaints such as nausea and vomiting, its reversibility gets more difficult in direct proportion to the time elapsed, its revision is more difficult than that of other methods, its restrictive effect remains insufficient, and it has also some other complications specific to it such as partial fold herniation and blockage. 


\section{Indications}

- Similar to sleeve gastrectomy

- BMI 35-45

- Volume eaters

- Revisions

- Band-Successful EWL

- RNYGB-Pouch Dilatation

- Sleeve-Dilatation

- Contraindications (relative)

- Metabolic syndrome - DM and dyslipidemia

- Reflux and hiatal hernia

- Super obese people

- Sweet eaters

- Snacks eaters

Gastric plication is not risk-free as all other operations. Possible complications:

- Leakage Rate is not exactly 0, a leakage has been reported.
- Bleeding - Short gastric or splenic veins; spleen

- Obstruction-Excessive plication or fold edema may obstruct the lumen.

- Nausea-vomiting- The most common complaint. Longtime, and sometimes requires IV treatment.

- GERD: Initially intense, and then up to the sleeve.

- Partial fold tear

- Gastric ulcer

- Necrosis

Intraoperative Complications:

- Bleeding from short gastric veins or spleen

- Thermal damage associated with Harmonic application

- Gastric rupture associated with tense suture

- Hematoma at the suture line

Postoperative Complications:

- Stenosis and internal blockage (Figure 7)
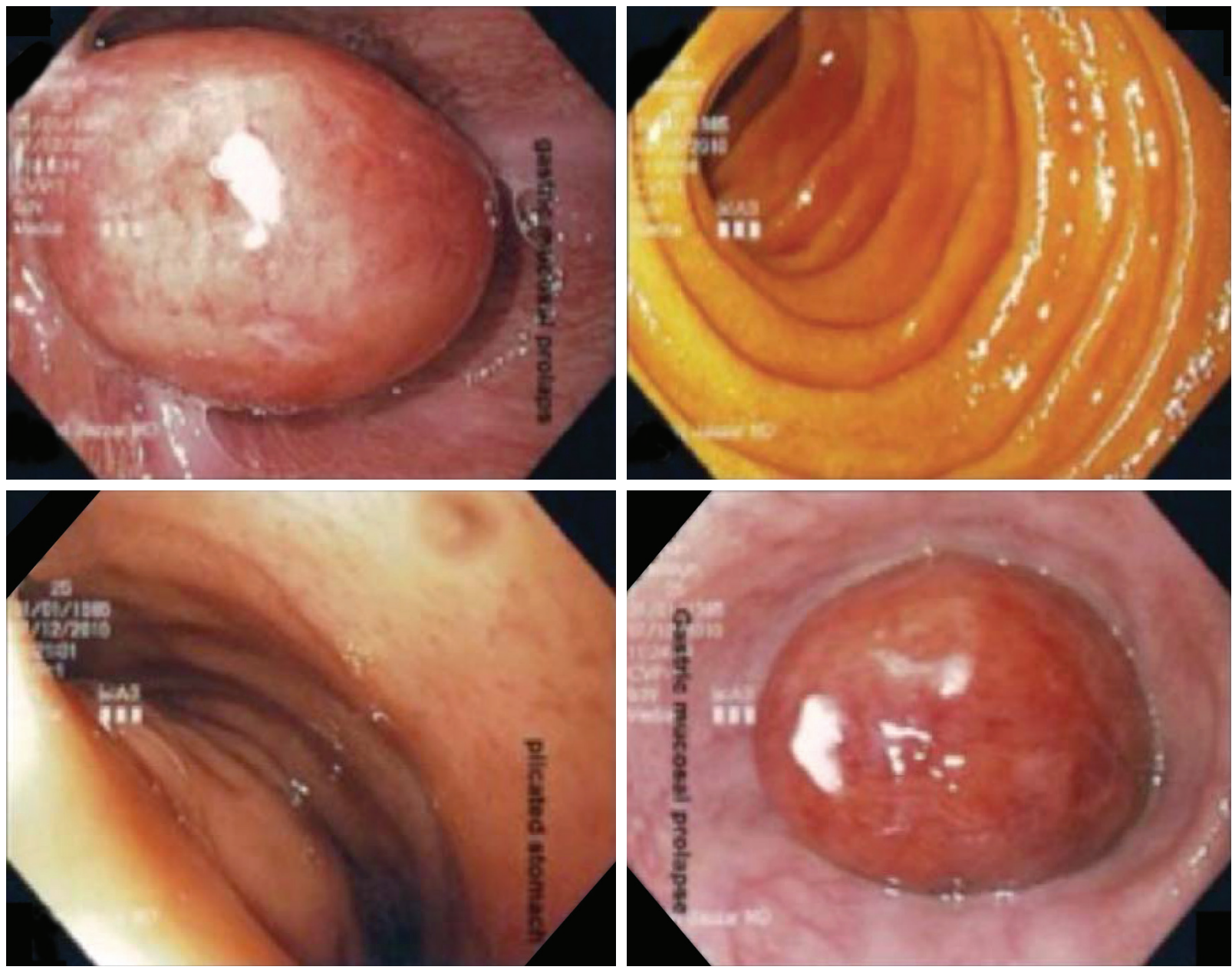

Figure 7. 
- Mucosal herniation at the suture line

- Despite being rare, leak

- GERD

- Nausea-vomiting

- ARF (acute renal failure) in case of inadequate IV replacement

When the currently available publications are examined, laparoscopic gastric plication seems to be a safe and effective method for the surgical treatment of morbid obesity. It has been reported to provide improvement in the lipid profile and metabolic values in association with weight loss. However, longer-term comparative studies on the amount and permanence of weight loss are required to be carried out. Likewise, long-term comparative studies are required for investigating the efficacy and safety. It can be offered as an option to the patient after giving him/ her the required detailed information; however, it should be noted that revision operations become very difficult to perform in parallel with the time elapsed. It should not be preferred for patients with reflux and hiatal insufficiency in particular since it will dramatically worsen their postoperative comfort in life.

\section{References}

1. Tsigos C, Hainer V, Basdevant A, Finer N, Fried M, Mathus-Vliegen E, et al; Obesity Management Task Force of the European Association for the Study of Obesity. Management of obesity in adults: European clinical practice guidelines. Obes Facts 2008;1:106-16.

2. Shields $M$, Tjepkema $M$. Trends in adult obesity. Health Rep 2006;17:53-9.

3. Calle EE, Rodriguez C, Walker-Thurmond K, Thun MJ. Overweight, obesity, and mortality from cancer in a prospectively studied cohort of U.S. adults. N Engl J Med 2003;348:162538.

4. Wing R, Phelan S. Science-Based Solutions to Obesity: What Are the Roles of Academia, Government, Industry, and Health Care? Boston, MA, March 10-11, 2004 and Anaheim, CA, October 2, 2004.

5. Rucker D, Padwal R, Li SK, Curioni C, Lau DC. Long term pharmacotherapy for obesity and overweight: updated meta-analysis. BMJ 2007;335:1194-9.

6. Jacobs M, Bisland W, Gomez E, Plasencia G, Mederos R, Celaya $\mathrm{C}$, et al. Laparoscopic sleeve gastrectomy: a retrospective review of 1- and 2-year results. Surg Endosc 2010;24:781-5.

7. Maggard MA, Shugarman LR, Suttorp M, Maglione M, Sugerman $\mathrm{HJ}$, Livingston $\mathrm{EH}$, et al. Meta-analysis: surgical treatment of obesity. Ann Intern Med 2005;142:547-59.

8. Santry HP, Gillen DL, Lauderdale DS. Trends in bariatric surgical procedures. JAMA 2005;294:1909-17.

9. Braghetto I, Korn O, Valladares H, Gutiérrez L, Csendes A, Debandi $A$, et al. Laparoscopic sleeve gastrectomy: surgical technique, indications and clinical results. Obes Surg 2007;17:1442-50

10. Picot J, Jones J, Colquitt JL, Gospodarevskaya E, Loveman E, Baxter $L$, et al. The clinical effectiveness and cost-effectiveness of bariatric (weight loss) surgery for obesity: a systematic review and economic evaluation. Health Technol Assess 2009;13:1-190.

11. Nocca D, Frering V, Gallix $B$, de Seguin des Hons $C$, Noël $P$, Foulonge MA, et al. Migration of adjustable gastric banding from a cohort study of 4236 patients. Surg Endosc 2005; 19:947-50.

12. Tretbar LL, Taylor TL, Sifers EC. Weight reduction. Gastric plication for morbid obesity. J Kans Med Soc 1976;77:488-90.

13. Wilkinson LH, Peloso OA. Gastric (reservoir) reduction for morbid obesity. Arch Surg 1981;116:602-5.

14. Talebpour M, Amoli BS. Laparoscopic total gastric vertical plication in morbid obesity. J Laparoendosc Adv Surg Tech A 2007; 17:793-8.

15. Brethauer SA, Harris JL, Kroh M, Schauer PR. Laparoscopic gastric plication for treatment of severe obesity. Surg Obes Relat Dis 2011;7:15-22.

16. Atlas H, Yazbek T, Garneau PY, Safa N, Denis R. Is there a future for Laparoscopic Gastric Greater Curvature Plication (LGGCP)? a review of 44 patients. Obes Surg 2013;23:1397-403.

17. Shen D, Ye H, Wang Y, Ji Y, Zhan X, Zhu J, et al. Comparison of short-term outcomes between laparoscopic greater curvature plication and laparoscopic sleeve gastrectomy. Surg Endosc 2013;27:2768-74.

18. El-Geidie A, Gad-el-Hak N. Laparoscopic gastric plication: technical report. Surg Obes Relat Dis 2014;10:151-4.

19. Talebpour M, Vahidi H, Talebpour A. Eleven years experience about the new technique "laparoscopic vertical gastric plication" in morbid obesity introduced the first time in the world. In: Proceeding of the XVI Congress of the International Federation for the Surgery of Obesity and Metabolic Disorders (IFSO) 2011;31:1017.

20. Saunders R. Post-surgery group therapy for gastric bypass patients. Obes Surg 2004;14:1128-31.

21. Ashton K, Heinberg L, Windover A, Merrell J. Positive response to binge eating intervention enhances postoperative weight loss. Surg Obes Relat Dis 2011;7:315-20. 